\title{
EVOLUCIÓN DE LOS PARÁMETROS ANTROPOMÉTRICOS Y CONDICIONALES EN DEPORTISTAS PERTENECIENTES AL ÁREA DE FÚTBOL JOVEN, EDADES COMPRENDIDAS ENTRE 12 Y 15 AÑOS. CLUB PROFESIONAL UNIVERSIDAD DE CONCEPCIÓN- CHILE
}

\author{
Cristián Eduardo Luarte, Universidad de San Sebastian - USS - Chile
}

\begin{abstract}
RESUMEN
El objetivo del presente estudio consistió en determinar los aspectos físicos y antropométricos en futbolistas entre 12 y 15 años. El estudio es de tipo no experimental descriptivo de corte transversal. Se estudiaron 76 sujetos sanos, practicantes de fútbol de sexo masculino con edades comprendidas entre los $12(n=19), 13(n=20), 14(n=18)$ y 15 años $(n=19)$ a quienes se les evaluó las variables antropométricas de peso corporal, estatura, pliegues cutáneos tricipital y subescapular y las cualidades físicas de flexibilidad, salto vertical con contra-movimiento (CMJ), velocidad en $30 \mathrm{~m}$ y la resistencia aeróbica, a través del test de carrera Naveta. Se encontraron diferencias significativas $(p<0,05)$ en los parámetros antropométricos y las cualidades físicas condicionales. La estatura, el peso corporal aumentan entre los 12 y 15 años, en tanto la masa grasa disminuye. La fuerza explosiva, la velocidad y la resistencia aumentan, en tanto la flexibilidad aumenta escasamente tendiendo a disminuir y a detenerse. Cuando se compararon los resultados de este estudio con otras investigaciones, se pudo observar que la fuerza explosiva, la velocidad, la resistencia y la flexibilidad, el rendimiento fue menor. Como conclusión se puede señalar que la edad cronológica afecta el rendimiento de los aspectos antropométricos y condicionales de la fuerza explosiva, la velocidad, la resistencia y la flexibilidad, pero de forma no exclusiva, ya que es necesario tomar en cuenta las diferencias individuales, el nivel de maduración biológica y la respuesta al entrenamiento y no solo agrupar a los deportistas por esta variable.
\end{abstract}

Palabras-Claves: Fútbol joven; Rendimiento físico; Parámetros antropométricos; Parámetros y condicionales

\section{EVOLUTION AND CONDITIONAL ANTHROPOMETRIC PARAMETERS IN AREA SPORTS FOOTBALL OUTSIDE THE YOUNG AGED 12 AND 15 YEARS. CLUB PROFESSIONAL UNIVERSITY OF CONCEPCION-CHILE.}

\section{ABSTRACT}

The objective of this study was to determine the physical and anthropometric Soccer Players between 12 and 15 years. The study is non-experimental cross-sectional descriptive. We studied 76 healthy subjects, soccer practitioners male sex with ages between $12(\mathrm{n}=19), 13$ $(\mathrm{n}=20), 14(\mathrm{n}=18)$ and 15 years $(\mathrm{n}=19)$ to whom evaluated anthropometric variables, body weight, height, triceps and subscapular skinfolds and physical qualities of flexibility, vertical jump with counter-movement (CMJ), speed $30 \mathrm{~m}$ and endurance through Naveta running 
test. There were significant differences $(\mathrm{p}<0.05)$ in anthropometric parameters and conditional physical qualities. Height, body weight increases between 12 and 15 years, while decreasing fat mass. Explosive strength, speed and endurance increases, while increasing slightly the flexibility tends to decrease and stop. When comparing the results of this study with other research, it was observed that explosive strength, speed, endurance and flexibility, performance was lower. In conclusion we can say that the chronological age affects the performance of anthropometric and conditional aspects of explosive strength, speed, endurance and flexibility, but not exclusively so, since it is necessary to take into account individual differences, the level of biological maturation and response to training and not just group the athletes for this variable.

Key words: Young soccer; Physical performance; Anthropometric parameters and conditional 


\section{INTRODUCCIÓN}

El deporte contemporáneo debe estar orientado hacia un campo de investigación y reflexión pedagógica, los juegos colectivos en general se caracterizan, de acuerdo a ciertas constantes; materiales, terrenos, reglas y progresión de los jugadores, en relación al adversario. ${ }^{1}$ A partir de características fisiológicas de deportistas pertenecientes al juego colectivo de invasión con adversarios; fútbol y de su naturaleza compleja, presentamos este estudio. Ello implica el control de un gran número de factores y variables. Sin embargo, es necesario señalar que los alcances del deporte en el reciente siglo, lo ubica como uno de los fenómenos socioculturales más representativos, con diversidad de significados, diversos escenarios y grupos sociales. ${ }^{2}$

El deporte es uno de los más importantes fenómenos socioculturales en la contemporaneidad y que es posible de ser observado por el creciente interés de la sociedad, por sus prácticas deportivas corporales. $^{2}$

Este estudio fue analizado desde una perspectiva biológica, específicamente desde la dimensión fisiológica, característica fundamental que debe poseer un futbolista y que debe identificarse en primer lugar, con las capacidades neuromusculares y, a continuación, con los sistemas bioenergéticas señalados para suministrar energía bioquímica, que se transforma en trabajo mecánico en los músculos. ${ }^{3}$ De igual forma, se abordaron indicadores biológicos e indicadores del comportamiento y desempeño motor. Estos son fundamentales en acompañar el crecimiento físico y sus variables fisiológicas.

Es importante señalar que son muchos los factores que influyen en el éxito deportivo de un futbolista, existiendo una gama de posibilidades y experiencias; motoras, sociales, culturales, afectivas y cognitivas que abordar. Sin embargo para este estudio, se ha considerado aspectos condicionales en las edades anteriormente señaladas.

El fútbol es un deporte colectivo de cooperación y oposición, se juega en forma simultánea en un espacio estandarizado y sus acciones son efímeras con un alto grado de incertidumbre. Desde el punto de vista de las capacidades físicas y antropométricas, tiene una participación intermitente durante el juego, expresado en las distintas acciones con y sin balón, como correr a distintas intensidades, correr en distintas direcciones, frenar, girar, saltar con uno o ambos pies y cualquier movimiento requerido de acuerdo a la situación de juego y generalmente en 
oposición. Las demandas propias del juego solicitan unas prestaciones que se enmarcan en un rango que está sobre sujetos sedentarios y los más elevados que caracterizan disciplinas en las que el rendimiento depende de la potencia aeróbica o de la fuerza explosiva o fuerza rápida $^{3}$. El juego del fútbol tiene una participación aeróbica de 70 - $75 \%$ y una participación anaeróbica del $20-25 \%{ }^{4-6}$

También la fuerza y la velocidad se encuentran solicitadas en el juego, es decir, la capacidad de una fortaleza de piernas desarrollada de acuerdo a las diferentes etapas biológicas de los futbolistas, apta para la producción de una alta potencia explosiva y capaz de esfuerzos cortos y muy intensos, utilizando mecanismos energéticos de tipo aláctico. Este mecanismo alactácido lleva implícita la utilización inmediata del ATP (Adenosín trifosfato) almacenado en el músculo, este permite un suministro solo de 2 o 3 segundos. $^{7}$ La flexibilidad es fundamental para realizar movimientos en cantidad y calidad, su óptimo desarrollo afecta las demás cualidades físicas y tiene influencia sobre el potencial de rendimiento existente, ${ }^{8}$ por otro lado las medidas antropométricas en el estudio morfológico de los deportistas, y su vinculación con la performance, parece hoy indiscutible, ${ }^{5}$ de la misma manera, algunas investigaciones han revelado que las características de la composición corporal son también un factor fundamental para el éxito competitivo en un deporte. ${ }^{9}$

El VO2 máx. es un parámetro que nos permite evaluar la capacidad de resistencia de un sujeto ante esfuerzos de baja intensidad y larga duración. Por lo anterior, se infiere que con el entrenamiento podríamos mejorar los niveles de este parámetro, entonces el deportista podría rendir más frete a este tipo de esfuerzo. ${ }^{10}$

Según Klissouras, los valores del VO2 vienen determinados en un $98 \%$ por factores hereditarios, por lo que los antecedentes familiares pueden ser un factor determinante del rendimiento en las pruebas de resistencia. ${ }^{10}$

Estudios efectuados nos indican que, valores que no superan los $60-65 \mathrm{ml} / \mathrm{min} / \mathrm{kg}$ en futbolistas, no representan valores demasiado elevados en comparación con otros deportes que requieren tener un predominio en el trabajo de resistencia. ${ }^{3}$ Sin embargo, debemos considerar que, una buena resistencia posibilita entre otras cosas, mantener a los deportistas disciplinados tácticamente y con un buen desempeño técnico. ${ }^{11}$ También es importante Conexões: revista da Faculdade de Educação Física da UNICAMP, Campinas, v. 12, n. 4, p. 1-22, out./dez. 2014. ISSN: $1983-9030$ 
considerar que la resistencia puede ser afectada por varios factores, como los fisiológicos, técnicos y biomecánicos. ${ }^{10}$

La resistencia se caracteriza por las posibilidades que tiene un deportista de realizar un trabajo muscular durante un tiempo prolongado, manteniendo los parámetros de movimiento cuyos niveles alcanzados son relativos al entrenamiento y a la herencia genética y los esfuerzos contemplan duraciones muy amplias, que van desde veinte segundos hasta seis horas o más. ${ }^{11}$

En función al parámetro condicional de velocidad, consideramos que, si bien esta característica neuromuscular está presente en diversas situaciones y en todos los deportes, en el caso del fútbol, el número de sprints realizados por los jugadores en un partido es elevado, sin embargo la duración de los mismos es reducida, aunque las acciones a máxima intensidad son minoritarias en números, su papel es importante para el desarrollo del juego, es decir, determinan el devenir del partido. ${ }^{8}$ Además, la velocidad representa una capacidad gregaria que depende en gran medida del trabajo de fuerza. ${ }^{12}$

Según Arruda e Cossio-Bolños, ${ }^{11}$ la velocidad representa la capacidad de un sujeto para realizar acciones motoras en un mínimo de tiempo y con un máximo de eficacia, en que las acciones motoras son desenvueltas con un máximo de velocidad y dentro de las circunstancias en un tiempo mínimo, en el supuesta que la tarea sea de corta duración y sin presentar cansancio.

Lo anterior, refuerza la idea de que la fuerza explosiva es fundamental, sin olvidar de que detrás existe una base de fuerza máxima. ${ }^{10}$ La fuerza explosiva hace referencia a fuerzas de desarrollo rápido, contra resistencias relativamente altas, en las que el deportista genera tensiones que aparecen rápidamente y aumentan gradualmente hasta el final del recorrido.

García Manso, J.; Navarro, M. y Ruiz, J. ${ }^{10}$ señalan que la fuerza explosiva es la capacidad del sistema neuromuscular de vencer una resistencia a la mayor velocidad de contracción posible. Harre y Hauptmann (1991) definen a esta cualidad como la capacidad de un atleta de vencer resistencias externas al movimiento con una gran velocidad de contracción. Esta cualidad de fuerza es la que permite al deportista imprimir a una masa una alta velocidad. 
La práctica del fútbol requiere de acciones de fuerza que afectan a la musculatura extensora de la rodilla y flexora de la cadera, en las cuales los movimientos requeridos son de tipo concéntrico, excéntrico e incluso isométrico. Por lo tanto, la musculatura de la parte posterior del muslo se limita a ejercer su acción antagónica ante esfuerzos explosivos, que en forma natural, es más preponderante en la musculatura anterior del muslo, esto por la gran utilización de las acciones de golpeo y pases durante el juego como acción agonística principal, por lo cual, la musculatura antagonista del muslo, se tiene que trabajar suficientemente y de manera específica, apuntando a la cualidad condicional de la capacidad de movimiento, ya que la limitación de movimiento en una articulación puede hipotecar la efectividad de un gesto deportivo y por consiguiente disminuir el rendimiento deportivo. ${ }^{10}$

Por su parte, la flexibilidad es importante en todos los deportes porque cualquier gesto deportivo tiene una amplitud y hay que tratar de que ésta no se vea reducida por la falta de desarrollo de dicha capacidad. ${ }^{13}$ De igual manera, podemos señalar que, la influencia de la flexibilidad en deportistas, obedece a características multifactoriales tales como; dimensiones psicológicas, fisiológicas, anatómicas, entrenamiento y factores ambientales. También podríamos considerar la influencia del sexo, edad, temperatura y periodo o estado del entrenamiento. ${ }^{11}$

Respecto a los jóvenes futbolistas, cabe señalar que las investigaciones son escasas en la región del Bío-Bío-Chile. Esto último motiva aún más este estudio, debido a que los jóvenes se encuentran en una etapa crucial de preparación y formación con vistas al alto rendimiento deportivo. Por lo tanto, es fundamental conocer y sistematizar la mayor cantidad y diversidad de información acerca de los jóvenes que son sometidos desde muy temprano a entrenamientos altamente especializados y sistemáticos en la búsqueda de mejores respuestas y mejores resultados en la competición.

La competición exagerada, cuyo foco principal es la victoria, reduce variadas posibilidades de deporte con un simple objetivo puntual. Hay un error cuando se reproduce la idea de que el deportista principalmente en las categorías de base, vale exclusivamente por sus resultados y de que todo se vincule con la competición. ${ }^{14}$

A esto se agrega otro problema, y es que los jóvenes - en el entrenamiento - son agrupados por edad cronológica, no siendo este un indicador fiable de la maduración biológica. ${ }^{15}$ 
Además, hay que considerar que la velocidad de los procesos de maduración psicobiológica y crecimiento físico es individual y distinta, por tanto las diferencias interindividuales pueden ser considerables. Por otro lado hay que mencionar que los jóvenes están expuestos a entrenamientos periódicos, lo que convierte al ejercicio en una variable a considerar, ${ }^{16}$ al concluir que el nivel de actividad puede influir en la composición corporal y no puede ser atribuida solamente a diferencias madurativas.

Los parámetros antropométricos, asociados con la composición corporal del futbolista deben ser un componente clave a evaluar durante el proceso de crecimiento y maduración, ya que sus cambios están asociados a la salud humana. ${ }^{11}$

El objetivo del presente estudio fue efectuar un diagnóstico de los aspectos físicos condicionales y antropométricos con el fin de estudiar el comportamiento de dichas variables entre los 12 y 15 años y determinar la existencia de diferencias significativas que pueden influir en el rendimiento del jugador.

\section{MÉTODOS}

El estudio fue de tipo no experimental de corte transversal y alcance descriptivo. ${ }^{17}$ Las evaluaciones antropométricas y físicas son evaluadas en su totalidad en un solo momento del tiempo. Todas las mediciones fueron efectuadas por profesores de Educación Física en las instalaciones del Estadio Deportivo Atlético Militar. Se utilizó una sala acondicionada para la medición de las medidas antropométricas, gimnasio para la evaluación de la flexibilidad, el salto horizontal, el salto vertical con contramovimiento (CMJ) y el test de Course Navette. En tanto, la velocidad se realiza en un campo de fútbol de pasto natural. Las evaluaciones se hacen durante la tarde en horario de 16:00 a 18:00horas, durante la última parte de la temporada del año 2012 coincidiendo con los horarios de entrenamiento del club. Se incluye a todos los deportistas inscritos en los registros del club y que tengan una antigüedad mínima de dos años. Los atletas extranjeros y los que presentan alguna lesión deportiva son excluidos. Se exige consentimiento informado y firmado por los encargados del cuerpo técnico del club para permitir realizar las evaluaciones durante el año 2012.

Conexões: revista da Faculdade de Educação Física da UNICAMP, Campinas, v. 12, n. 4, p. 1-22, out./dez. 2014. 


\subsection{Objetivos}

Determinar los parámetros antropométricos y condicionales de futbolistas jóvenes y su relación con la edad cronológica comprendida entre los 12 y 15 años.

Analizar la correlación existente entre los parámetros físicos, antropológicos y su edad cronológica.

\subsection{Muestra}

No probabilística, intencionada. Se estudiaron 76 sujetos sanos, practicantes de fútbol de sexo masculino con edades comprendidas entre los $12(n=19), 13(n=20), 14(n=18)$ y 15 años ( $n=19)$. Los sujetos se encontraban finalizando el periodo competitivo del presente año, el cual comprende la realización de microciclos de cuatro días por semana, con una duración de 90 minutos por día para la edad de 12 a 15 años. La población de estudio presentó una media de edad de 13,49 \pm 1,13 años, peso corporal (PC) de 55,63 \pm 10,69 kg., estatura de $161,86 \pm 10,23 \mathrm{~cm}$., porcentaje de grasa corporal $(\% \mathrm{G})$ de $12,82 \% \pm 3,14 \%$.

\subsection{Evaluación antropométrica}

En cuanto a las mediciones antropométricas se sigue las normas y recomendaciones descritas por la Sociedad Internacional para el avance de la Cineantropometría (ISAK). ${ }^{18}$ Las mediciones antropométricas se realizan por triplicado, con aproximadamente un minuto de separación entre ellas, en ropa interior y posición de bipedestación.

Para determinar la masa corporal total se utiliza una balanza digital de marca Tanita con una precisión de $1 \mathrm{k}$ con una escala de 0 a $140 \mathrm{~kg}$. Se determina la estatura del individuo en posición erecta, mediante una cinta métrica metálica no extensible de marca Lufkin graduada en $\mathrm{cm}$ con una escala de 0 a $200 \mathrm{~cm}$. Se determinan los pliegues cutáneos de tríceps y subescapular utilizando un compás de marca Slim Guide con una precisión de 1 milímetro en el lado no dominante del sujeto. La lectura se realiza a los 4 segundos y todas las determinaciones son realizadas por triplicado y por el mismo observador. El cálculo del porcentaje de grasa se realiza mediante la ecuación de regresión propuesta por Slaughter, la 
cual es descrita en Heyward ${ }^{19}$ y Fernandes Filho, ${ }^{20}$ para muchachos blancos; $\% \mathrm{G}=1,21\left(\Sigma_{2}\right)$ $-0,008\left(\Sigma_{2}\right)^{2}-3,4$ (12 a 14 años $)-\% G=1,21\left(\Sigma_{2}\right)-0,008\left(\Sigma_{2}\right)^{2}-5,5$ (15 a 18 años $)$.

\subsection{Evaluación de las cualidades físicas}

En cuanto a las pruebas físicas, éstas se realizaron conforme a las recomendaciones y protocolos estandarizados a nivel internacional y nacional. La batería de test físicos comprende el test de flexibilidad de Wells y Dillon adaptado, el test de salto Horizontal (SH), el test de salto vertical con contramovimiento (CMJ), el test de velocidad de 30 metros planos, el test de Course Navette.

Las pruebas físicas fueron realizadas el mismo día y se evaluaron tres veces con la excepción del test de Course Navette. Se respeta el siguiente orden de aplicación de las pruebas: Flexibilidad, salto horizontal, salto vertical, velocidad y resistencia aeróbica. Antes de realizar las pruebas, los individuos efectúan ejercicios de calentamiento para la entrada en calor.

El test de flexibilidad mide la movielasticidad de la región dorso-lumbar e isquiotibial, a través de una posición de sentarse y alcanzar utilizando un equipamiento de madera para su medición. Se utiliza una escala de 0-50 cm, siguiendo el procedimiento adaptado conforme a las recomendaciones del Ministerio de Educación ${ }^{21}$ y Martínez. ${ }^{22}$ Se registra el mayor valor tras tres intentos.

La fuerza explosiva del tren inferior se mide a través de dos pruebas; el test de salto horizontal (SH), que evalúa la fuerza explosiva del tren inferior a partir de un salto horizontal. Se mide con una cinta métrica de nylon con una precisión de $0,1 \mathrm{~cm}$, siguiendo las recomendaciones de Blázquez. ${ }^{23}$ Debiendo realizar el jugador un impulso con ambas piernas hacia delante, acompañado de un balanceo de brazos. Se registra el mejor de tres intentos.

El segundo test utilizado es el test de salto vertical con contramovimiento (CMJ), que evalúa la fuerza explosiva del tren inferior a partir de un salto vertical con contramovimiento. Se siguen las recomendaciones efectuadas por Bosco. ${ }^{3}$ El salto vertical se evalúa, a través de un 
sistema de evaluación cinemática con plataforma de saltos de marca Axón Jump conectada a un computador marca HP con utilización de un programa en CD-ROOM de autoría del fabricante Axón Jump. Después de tres intentos con una pausa de 40 segundos, se anota el mejor valor alcanzado en $\mathrm{cm}$.

La velocidad se determina a través de una carrera de 30 metros con partida alta siendo activado el cronómetro en el momento que el ejecutante dio el primer paso de salida. Se utiliza un cronómetro digital de marca Casio ${ }^{\circledR}(1 / 100 S e g)$. Después de tres intentos se anota el mejor tiempo registrado en segundos y centésimas. Se siguen las recomendaciones hechas por Martínez. ${ }^{22}$ Entre cada intento se establece una pausa completa para cada individuo.

Por último, se utiliza el test progresivo de carrera naveta, que es un test de potencia aeróbica que evalúa la resistencia cardiovascular. Se realiza mediante una carrera de ida y vuelta en un espacio de $20 \mathrm{~m}$ tipo naveta, utilizando un pendrive de $4 \mathrm{~g}$ de marca Verbatim con ajuste de señal auditiva (bip), conectado vía puerto USB a un equipo BT-351525 casa Royal. Se adoptaron las recomendaciones hechas por el Mineduc. ${ }^{21}$ Se considera como resultado final el minuto de carrera de abandono de la prueba y con ello se determina de forma indirecta el consumo máximo de oxígeno (VO2máx.), utilizando la ecuación $(\mathrm{y}=-27,4+6 \mathrm{x})$, donde $\mathrm{x}$ es la velocidad de carrera en $\mathrm{K} / \mathrm{h}$.

\subsection{Análisis estadístico}

Se utiliza la media aritmética (X) y la desviación estándar (DE) como medidas principales de la estadística descriptiva. Para el análisis de la distribución normal de la muestra se utiliza la prueba de normalidad de Kolmogorov-Smirnov. Para verificar las diferencias significativas entre las edades de cada una de las variables se utiliza ANOVA de un factor y la prueba de especificidad de Tukey $(p<0,05)$. La estabilidad de las pruebas se evalúa mediante el error técnico de medida (ETM) y la reproductibilidad se determina con el coeficiente de correlación de Pearson ( $r$ ) con una probabilidad de $p<0,01$. Las variables antropométricas arrojan un error técnico de medida (ETM) inferior a 0,5\% y un coeficiente de reproductividad de $r=0,99$. Las variables físicas muestran valores inferiores al $4 \%$ y un nivel de reproductividad de $\mathrm{r}=0,94$ a 0,99 , respectivamente. Para controlar la confiabilidad 
de las medidas antropométricas y físicas se evalúa en dos oportunidades (test-retest) a un grupo de 20 sujetos en un mismo día, se sigue dicho procedimiento cada 5 evaluaciones. En tanto, para el test de Course Navette, el re-test se realiza después de 72 horas en las mismas condiciones. Se utilizó el programa estadístico SPSS 15.0 para Windows.

\section{RESULTADOS}

Los valores medios referidos al peso corporal expresados en la Figura 1, corresponden a 43,78 \pm 6,67 para 12 años, 53,85 $\pm 6,86$ para 13 años, $61,66 \pm 8,59$ para 14 años y 63,63 \pm 7,52 para 15 años. Al contrastar los grupos de edades, se aprecia que entre el grupo de 14 años y 15 años no hay diferencias significativas $(\mathrm{p}=0,852)$, a su vez en todas los demás comparaciones múltiples se visualizan diferencias significativas, 12/13 años $(\mathrm{p}=0,000)$, $12 / 14$ años $(\mathrm{p}=0,000), 12 / 15$ años $(\mathrm{p}=0,000), 13 / 14$ años $(\mathrm{p}=0,010)$ y $13 / 15$ años $(\mathrm{p}=$ $0,001)$.

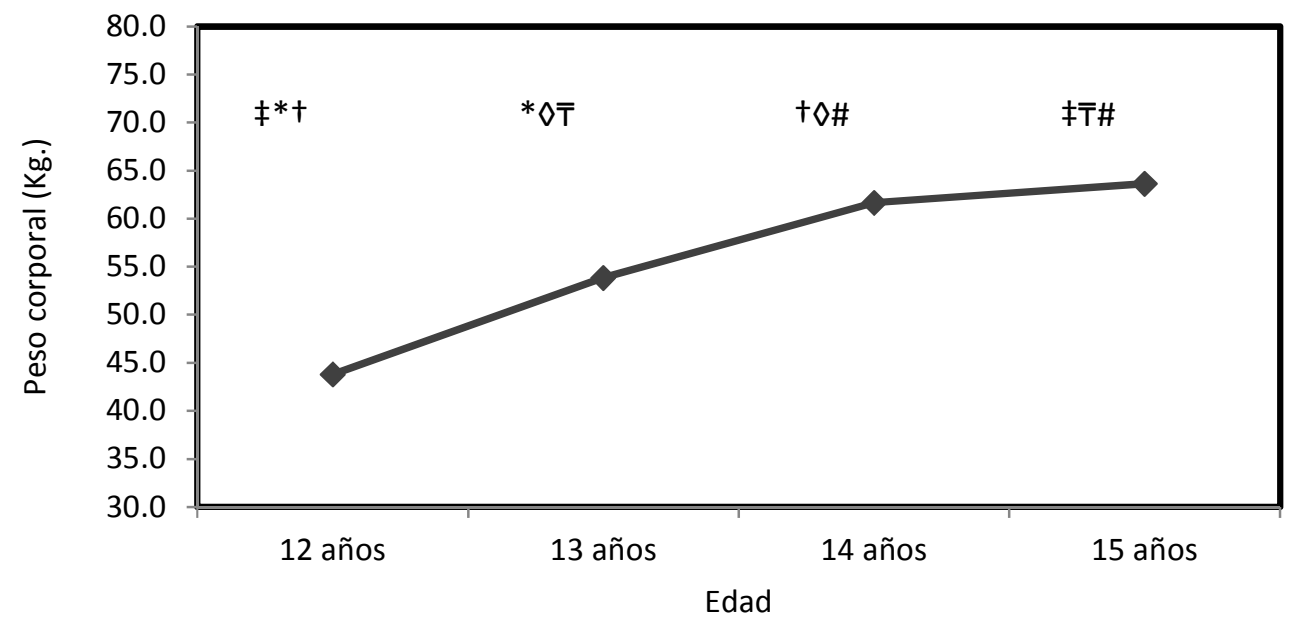

Figuras1: Manifestación del peso corporal según edad.

$$
\text { ( } * p=0,000 ; \uparrow p=0,000 ; \neq p=0,000 ; \diamond p=0,010 ; \bar{T} p=0,001 ; \# p=0,852 \text { ) }
$$

La estatura presenta una tendencia creciente (FIGURA 2), manifestada desde los 12 años con valores medios de 150,10 \pm 8,17, 162,50 \pm 6,13 (13 años), 166,13 $\pm 8,74$ (14 años) y 168,89 $\pm 6,41$ (15 años). Se aprecian diferencias significativas entre 12/13 años $(p=0,000), 12 / 14$ 
años $(\mathrm{p}=0,000), 12 / 15$ años $(\mathrm{p}=0,000)$ y $13 / 15$ años $(\mathrm{p}=0,043)$, por el contrario no se observan cambios significativos entre $13 / 14$ años $(p=0,437)$ y 14/15 años $(p=0,672)$.

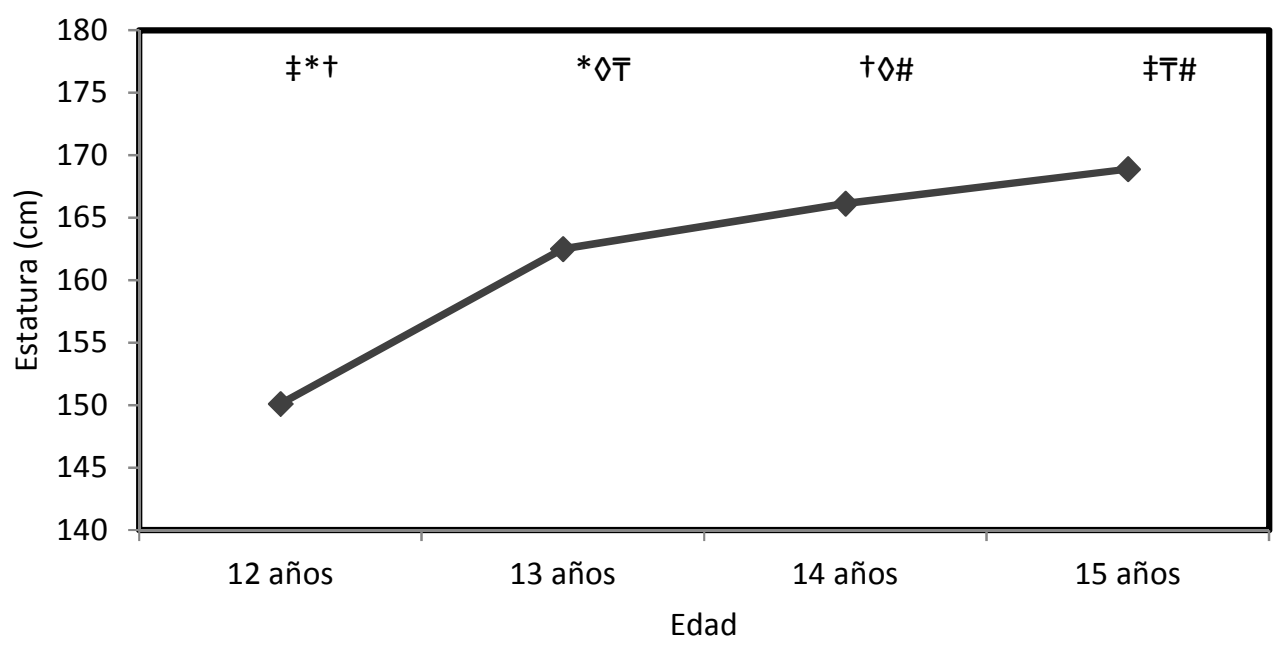

Figura 2: Evolución de la estatura corporal de acuerdo a nivel etáreo.

$$
(* p=0,000 ;+p=0,000 ; \ddagger p=0,000 ; \diamond p=0,437 ; \bar{T} p=0,043 ; \# p=0,672)
$$

Los valores medios expresados en porcentajes de la masa grasa observados en la figura 3 , corresponden a 14,26 $\pm 3,67$ en el grupo de 12 años, 13,36 $\pm 3,45$ en el grupo de 13 años, $12,24 \pm 2,07$ en los jóvenes de 14 años y 11,34 $\pm 2,42$ en los de 15 años respectivamente. Se aprecia que dichos valores disminuyen conforme avanza la edad pero sin diferencias significativas en todas las comparaciones, $12 / 13$ años $(p=0,787), 12 / 14$ años $(p=0,180)$, 13/14 años ( $\mathrm{p}=0,658), 13 / 15$ años $(\mathrm{p}=0,162)$ y 14/15 años ( $\mathrm{p}=0,800)$, salvo entre 12 y 15 años donde se obtiene un valor $(\mathrm{p}=0,019)$ que constituye una diferencia significativa. 


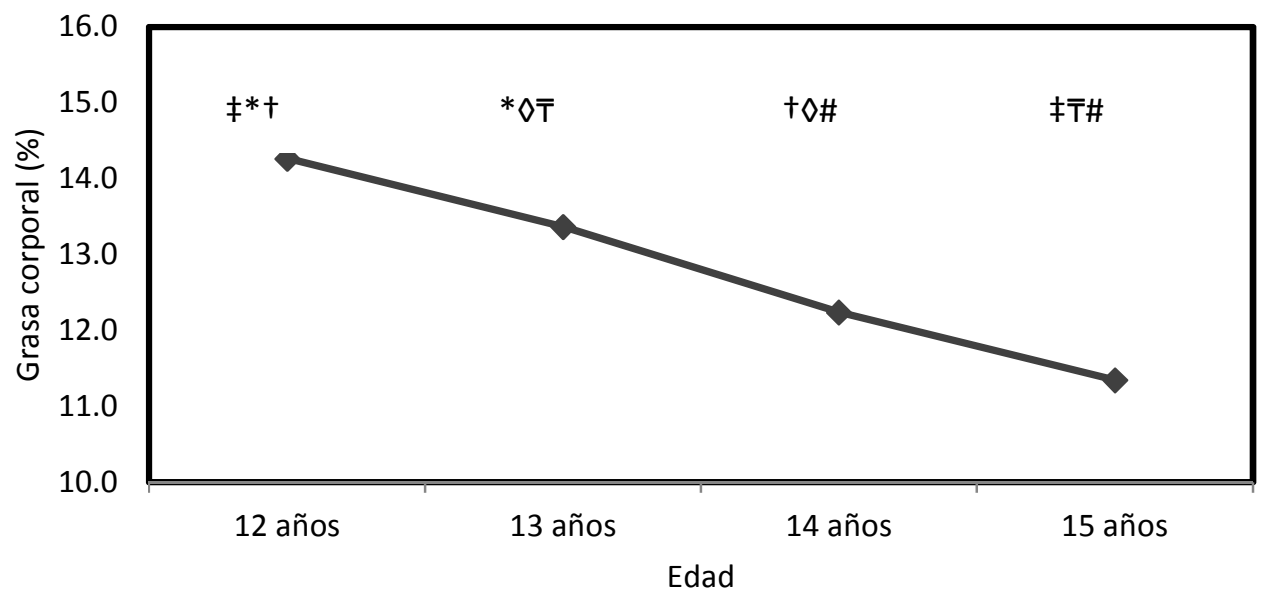

Figura 3: Valores medios de la masa grasa corporal por rangos de edades.

$$
\text { ( } * p=0,787 ; \uparrow p=0,180 ; \neq p=0,019 ; \diamond p=0,658 ; \bar{T} p=0,162 ; \# p=0,800 \text { ) }
$$

La Figura 4 muestra la evolución de la flexibilidad entre los 12 y los 15 años, los valores expresados en media y desviación estándar son de 25,84 \pm 3,46 para el grupo de 12 años, $29,10 \pm 3,40$ para 13 años, $32,00 \pm 4,27$ para 14 años y 34,68 $\pm 5,75$ para 15 años. Se observan diferencias significativas al comparar los grupos 12/14 años $(\mathrm{p}=0,000), 12 / 15$ años $(\mathrm{p}=$ $0,000)$ y $13 / 15$ años $(\mathrm{p}=0,001)$, por el contrario no se aprecian diferencias significativas entre los grupos 12/13 años ( $p=0,095), 13 / 14$ años $(p=0,174), 14 / 15$ años $(p=0,214)$.

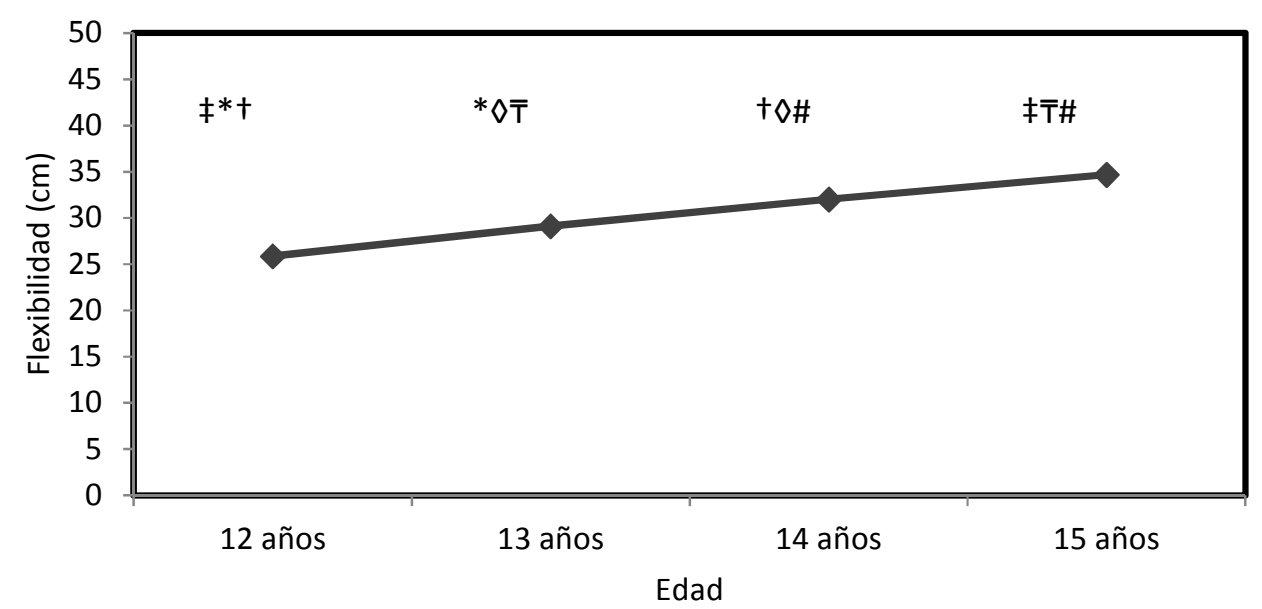

Figura 4: Valores medios de la flexibilidad a partir de los 12 años.

$(* p=0,095 ;+p=0,000 ; \ddagger p=0,000 ; \diamond p=0,174 ; \bar{T} p=0,001 ; \# p=0,214)$ 
La fuerza explosiva evaluada a través del salto vertical con contramovimiento, tiene un incremento paulatino que se observa en la Figura 5, desde los 12 hasta los 15 años, encontrándose diferencias significativas en 12/13 años ( $p=0,001), 12 / 14$ años $(p=0,000)$, $12 / 15$ años $(p=0,000)$ y 13/15 años $(p=0,006)$. Esta evidencia sugiere que a partir de los 15 años se producen valores más altos en el desarrollo de la fuerza explosiva contrariamente a lo que sucede antes de los 15 años donde no hay ganancias importantes en fuerza explosiva, por lo tanto, no hay diferencias significativas en $13 / 14$ años $(p=0,594)$ y 14/15 $(p=0,165)$. Los valores medios encontrados son 25,92 $\pm 2,55$ para 12 años, 29,79 \pm 3,89 para 13 años, $31,05 \pm 2,89$ para 14 años y $33,18 \pm 2,79$ para 15 años.

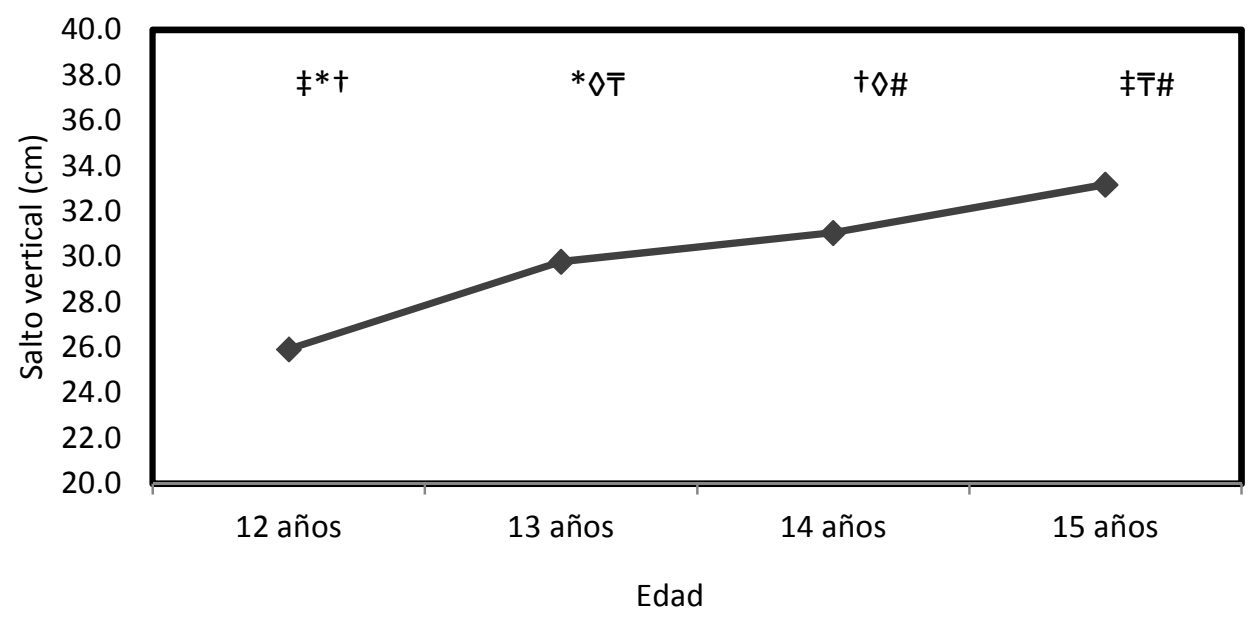

Figura 5: Valores medios de la fuerza explosiva vertical con contramovimiento.

$$
(* p=0,001 ;+p=0,000 ; \neq p=0,000 ; \diamond p=0,594 ; \bar{\top} p=0,006 ; \# p=0,165)
$$

La velocidad de $30 \mathrm{~m}$ da cuenta (figura 6) de valores medios de 5,00 \pm 0,25 para 12 años, 4,70 $\pm 0,18$ para 13 años, 4,55 $\pm 0,23$ para 14 años y 4,40 $\pm 0,13$ para 15 años. Se observa una disminución del tiempo de recorrido conforme aumenta la edad desde los 12 años hasta los 15 años. En concordancia con el análisis estadístico realizado se observan diferencias significativas con respecto a 12/13 años ( $\mathrm{p}=0,000), 12 / 14$ años $(\mathrm{p}=0,000), 12 / 15$ años $(\mathrm{p}=$ $0,000)$ y $13 / 15$ años $(p=0,000)$. Por su parte en los contrastes $13 / 14$ años $(p=0,133)$ y 14/15 años $(p=0,109)$ no se encontraron diferencias significativas. 


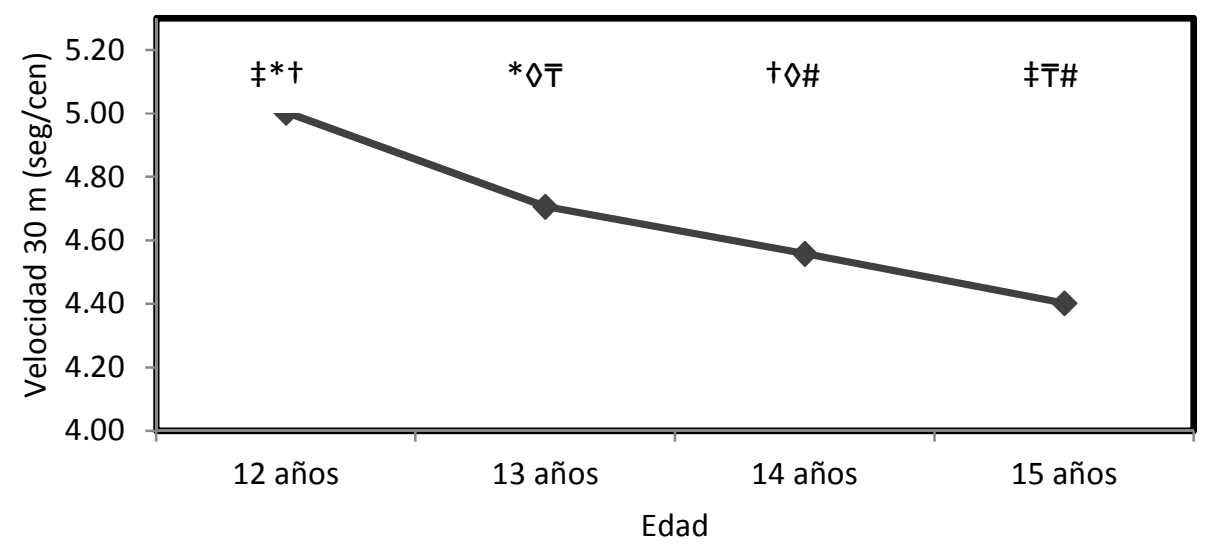

Figura 6: Valores medios en segundos y centésimas de la velocidad en 30 metros.

$$
\text { ( } * p=0,000 ; \uparrow p=0,000 ; \neq p=0,000 ; \diamond p=0,133 ; \bar{T} p=0,000 ; \# p=0,109 \text { ) }
$$

El comportamiento del consumo máximo de oxígeno expresado en $\mathrm{ml} / \mathrm{kg} / \mathrm{min}$ obtenido indirectamente a través del test de carrera Naveta se incrementa hasta los 14 años, luego se estabiliza y permanece estable sin evidenciar diferencias significativas hasta los 15 años, como puede observarse en la figura 7, los valores medios encontrados dan cuenta de 55,34 \pm 3,39 para 12 años, 57,91 $\pm 4,03$ para 13 años, 60,64 $\pm 2,47$ para 14 años y de 59,29 $\pm 3,86$ para 15 años. En las comparaciones múltiples post hoc de Tukey se observan solamente dos diferencias significativas, $12 / 14$ años $(\mathrm{p}=0,000)$ y 12/15 años $(\mathrm{p}=0,006)$. Las demás comparaciones no arrojan diferencias estadísticamente significativas, 12/13 años ( $\mathrm{p}=0,119)$, 13/14 años $(\mathrm{p}=0,119), 13 / 15$ años $(\mathrm{p}=0,628)$ y 14/15 años $(\mathrm{p}=0,700)$.

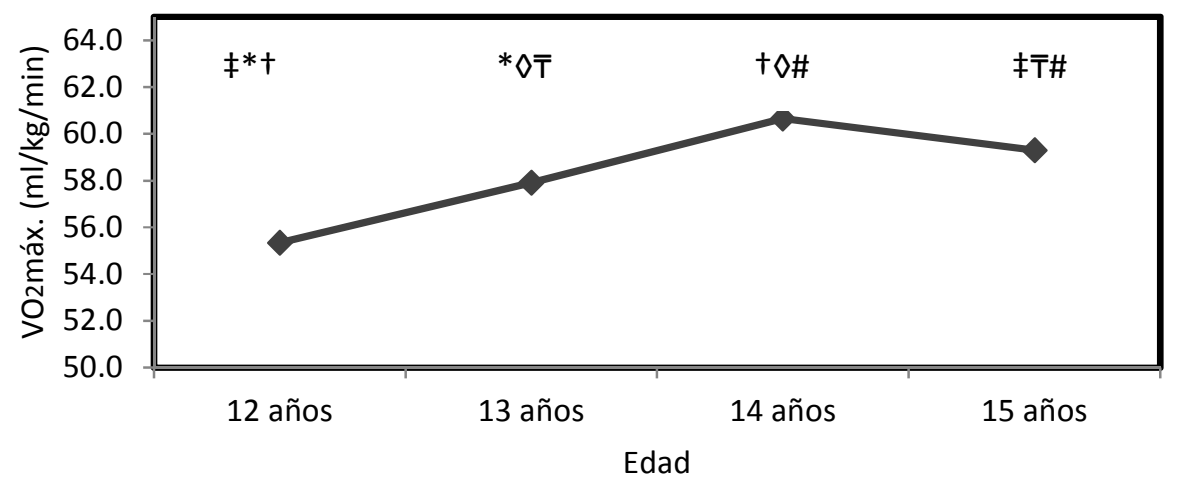

Figura 7: Comportamiento del consumo máximo de oxígeno expresado en $\mathrm{ml} / \mathrm{kg} / \mathrm{min}$.

$$
\left({ }^{*} p=0,119 ;+p=0,000 ; \neq p=0,006 ; \diamond p=0,119 ; \bar{\top} p=0,628 ; \# p=0,700\right)
$$




\section{DISCUSIÓN}

$\mathrm{Al}$ analizar los resultados de este estudio queda en evidencia que el peso corporal y la estatura aumentan gradualmente desde los 12 a los 15 años y en sentido inverso la masa grasa disminuye conforme avanza la edad. El ritmo de crecimiento en longitud se incrementa pero al mismo tiempo se ralentiza de forma exponencial con la edad, pero que es interrumpida en el período de la pubertad donde es posible observar incrementos de $15 \mathrm{~cm}$ en un período de dos años, aunque en este periodo puberal las acciones de crecimiento biológico están influenciadas por las hormonas reproductivas al superponerse sobre las acciones de la hormona de crecimiento. ${ }^{15}$ El peso corporal aumenta en este período y la masa grasa disminuye en forma proporcional según avanza la edad, en la fase puberal se produce una aceleración de la masa magra con un aumento del peso corporal y mediado también por la actividad física desarrollada. ${ }^{15} \mathrm{Al}$ comparar nuestros resultados con un estudio de jugadores brasileños, ${ }^{24}$ nos damos cuenta que el comportamiento de las variables es similar aunque nuestros valores son menores en las variables peso corporal y estatura y superior en masa grasa, aunque nuestros valores se encuentran en rangos normales para nuestra población, no así con la población comparada, por lo tanto, los aspectos nutricionales y genéticos podrían ser variables importantes a considerar. Las cualidades físicas condicionales siguen un patrón de crecimiento normal, ${ }^{15}$ es decir, se observa aumento del consumo máximo de oxígeno, de la fuerza explosiva de las extremidades inferiores y un mejoramiento de los tiempos registrados en velocidad desde los 12 a los 15 años.

La flexibilidad aun cuando presenta valores que se incrementan paulatinamente, en estas edades, no constituyen cambios significativos, por el contrario esta cualidad tiende a estancarse y disminuir con el tiempo. Si se observa el comportamiento del $\mathrm{VO}_{2}$ máx. relativo, como indicador de la resistencia medida, a través del Test Naveta, éste no expresa cambios significativos entre las edades superiores de este estudio (13 a 15 años), lo que supone que otros factores como tipo de entrenamiento, nivel de maduración y el tamaño corporal podrían afectar su rendimiento, en ese sentido el presente estudio tiene resultados similares ${ }^{25-26}$ referido a un incremento importante del consumo máximo de oxígeno hacia los 14 años, que coincide con el pico de velocidad de crecimiento en esa etapa y que resulta significativa en

Conexões: revista da Faculdade de Educação Física da UNICAMP, Campinas, v. 12, n. 4, p. 1-22, out./dez. 2014. 
ese tramo y también considerar que nuevamente se presenta el mismo fenómeno, edades iniciales de este estudio tienen diferencias significativas con edades superiores.

La fuerza explosiva sigue un patrón de crecimiento desde los 12 a los 15 años respectivamente. Se observa que el mejor rendimiento está en los 15 años. Al comparar con estudios similares, ${ }^{24}$ los resultados de este estudio son inferiores pero se concuerda en que la fuerza explosiva tiene una tendencia de crecimiento de acuerdo a la edad cronológica, lo que también se aprecia en el estudio ${ }^{27}$ con niños futbolistas de 7 a 14 años. Por otro lado, se evidencian diferencias significativas en todas las edades estudiadas al evaluar niños de 7 a 16 años, ${ }^{28}$ y atribuye dichas diferencias a factores nutricionales, nivel socioeconómico y al tipo de entrenamiento, variables que no fueron abordadas en este estudio pero que sin embargo concuerda en que existen diferencias significativas en las edades estudiadas respecto a la variables cualidades físicas y antropométricas.

La tendencia general indica que la flexibilidad disminuye con la edad, ${ }^{2}$ por el contrario donde mejor se desarrolla es en la infancia y la adolescencia, siendo en la adolescencia donde se han encontrado mejores resultados, ${ }^{27-29}$ flexibilidad aumenta con la edad en esta población estudio, pero no es un cambio significativo, la tendencia observada tiene más significado al plantear un deterioro y estancamiento conforme avanza la edad. Sólo presenta diferencias significativas cuando se comparan las edades de 12 y 13 años con 15 años. En ese sentido el presente estudio concuerda con otra investigación en este tema, ${ }^{30}$ al indicar que no hay un desarrollo óptimo de esta cualidad física debido a que las orientaciones del entrenamiento en este nivel carecen de dirección hacia el alto rendimiento deportivo, tal como se expresa en varios estudios. ${ }^{24,27-28}$

La velocidad de desplazamiento en $30 \mathrm{~m}$ tiene un comportamiento en la cual el tiempo disminuye según avanza la edad cronológica, lo que implica que hay un mejoramiento de esta cualidad al analizar los resultados en cada intervalo de edad. Se observa que entre los 12 y los 13 años es donde se producen las mayores diferencias en comparación con las edades de 14 y 15 años. Los resultados muestran valores medios inferiores si se comparan con otros estudios, ${ }^{24}$ lo que implica un menor desarrollo de esta cualidad en términos generales y que 
puede tener varios factores intervinientes como los cambios en el ritmo de crecimiento, la maduración, la estimulación adecuada en cada nivel de desarrollo y la respuesta al entrenamiento que siempre es individual y por consiguiente debe respetar los principios biológicos y pedagógicos del entrenamiento deportivo, e ir acompañado de una correcta planificación de las cargas de entrenamiento, sobre todo en las edades iniciales de este estudio (12 y13años), ${ }^{8}$ al acentuar la importancia del entrenamiento de la velocidad en estas etapas, permitiendo una transformación de las fibras intermedias hacia las fibras rápidas (FT) y no como sucede a menudo en que las fibras intermedias se transforman en fibras lentas (ST), esto por un exceso de trabajo a baja intensidad y larga duración, tan propio en nuestro medio. Todos los valores medios por cada intervalo de edad, son menores a otros estudios ${ }^{24} \mathrm{y}$ superiores a otros. ${ }^{30}$

Actualmente existe una progresiva carrera por obtener un resultado precoz del deportista sin respetar su maduración biológica y la relación existente con su edad cronológica. Por tanto, los resultados de esta investigación, podrían contribuir a balancear las cargas de entrenamiento en jóvenes futbolistas, entendiendo a estas como una capacidad compleja e individual que ayuda en la adaptación de ciertos volúmenes de trabajo que determinan ajustes morfológicos, orgánicos y funcionales, sin que el organismo sufra daños, evitando el riesgo en la salud. ${ }^{24}$

Es necesario revisar continuamente el proceso de formación de futbolistas jóvenes y considerar todos los factores de comportamiento del juego, ${ }^{31}$ como los constitucionales, físicos, técnicos, tácticos y psicológicos, como también el aspecto nutricional y los factores del entorno, para su desarrollo en las distintas etapas de su formación. El fútbol requiere fuerza explosiva, resistencia aeróbica y anaeróbica, velocidad, agilidad y flexibilidad, ${ }^{8}$ elementos fundamentales para competir y tener una estabilidad en el rendimiento a lo largo del proceso sin presencia de lesiones que impidan llegar al alto rendimiento deportivo. Los profesores/entrenadores tienen la obligación de conocer en profundidad lo anterior para exigir respuestas motrices idóneas por parte de los jóvenes futbolistas cautelando el normal desarrollo de cada uno con la firme convicción de formar para el alto rendimiento deportivo 
y no entrenar para el rendimiento mediático alterando el proceso y favoreciendo el resultado por sobre la formación, sobre todo en etapas iniciales.

\section{CONCLUSIÓN}

Los resultados de este estudio permiten apreciar que la fuerza explosiva, la velocidad y la resistencia tienden a mejorar según avanza la edad cronológica desde los 12 a los 15, pero no de manera significativa en todas las edades estudiadas, por lo tanto, la edad cronológica no es el único factor a considerar. Por su parte, la flexibilidad tiene un comportamiento inverso, ya que disminuye conforme avanza la edad. Al comparar los resultados con otras investigaciones, en general se aprecian valores inferiores en todas las pruebas. Finalmente, se puede concluir que los aspectos condicionales y antropométricos se ven influenciados con la edad cronológica y por lo tanto, es un elemento importante a considerar, no siendo el único, dentro del proceso de entrenamiento deportivo de jóvenes futbolistas, además hay que señalar que los futbolistas son agrupados por edad cronológica. Entonces, es necesario tomar en cuenta las diferencias individuales, el nivel de maduración biológica y la respuesta al entrenamiento como medidas básicas para una correcta programación de las cargas de trabajo, además, hay que considerar otros factores como la alimentación, el autocuidado y la recurrencia de lesiones, sobre todo entre los 12 y 14 años, que es una etapa sensible de alta respuesta al entrenamiento.

\section{REFERÊNCIAS}

${ }^{1}$ REVERDito, R.; SCAGLIA, A. Pedagogia do esporte: jogos coletivos de invasão. São Paulo: Phorte, 2009.

${ }^{2}$ MONTAGNER, P. C.; REVERDITO, R.; SCAGLIA, A. J. Pedagogia do esporte: aspectos conceituais da competição e estudos aplicados. São Paulo: Phorte, 2013.

${ }^{3} \mathrm{BOSCO}, \mathrm{C}$. Aspectos fisiológicos de la preparación física del futbolista. Barcelona: Paidotribo, 1996.

Conexões: revista da Faculdade de Educação Física da UNICAMP, Campinas, v. 12, n. 4, p. 1-22, out./dez. 2014. 
${ }^{4}$ MAZZA, J. C. et al. Futbolista sudamericano de elite: morfología, análisis del juego y performance. Buenos Aires: Biosystem Servicio Educativo, 1995.

${ }^{5}$ REILLY T.; CABLE N.T.; RIENZI, E. G. Aptitud física y entrenamiento en el fútbol. Ed. Biosystem Servicio, 1998.

${ }^{6}$ BANGSBO, J. Entrenamiento de la condición física en el fútbol. Barcelona: Paidotribo, 2000.

${ }^{7}$ GROSSER, M.; BRUGGEMANN, P.; ZINTL, F. Alto rendimiento deportivo: planificación y desarrollo. Barcelona: Martínez Roca, 1989.

${ }^{8}$ WEINECK, E. J. Fútbol total: el entrenamiento físico del futbolista. Barcelona: Paidotribo, 1999.

${ }^{9}$ SHEPHARD, K.; ASTRAND, P. La resistencia en el deporte. Barcelona: Paidotribo, 1998.

${ }^{10}$ GARCÍA MANSO, J. M.; NAVARRO, M.; RUIZ, J. A. Bases teóricas del entrenamiento deportivo: principios y aplicaciones. Madrid: Gymnos, 1996.

${ }^{11}$ ARRUDA, M.; COSSIO-BOLAÑOS, M. Treinamento para jovens futbolistas. São Paulo: Phorte, 2010.

${ }^{12}$ COMETTI, G. Los métodos modernos de musculación. Barcelona: Paidotribo, 1999.

${ }^{13}$ BLUM, B. Los estiramientos. Barcelona: Hispano Europea, 1998.

${ }^{14}$ MONTAGNER, P. Intervenções pedagógicas no esporte: práticas e experiencias. São Paulo: Phorte, 2011.

Conexões: revista da Faculdade de Educação Física da UNICAMP, Campinas, v. 12, n. 4, p. 1-22, out./dez. 2014. 
15 RUIZ-PÉREZ, L.; GUTIERREZ, M.; GRAUPERA, J.; LINAZA, J.; NAVARRO, F. Desarrollo: comportamiento motor y deporte. Madrid: Síntesis, 2007.

${ }^{16}$ PARIZCOVA, J. Body fat and physical fitness. The Hague: Martinus Nijhoff, 1977.

${ }^{17}$ HERNÁNDEZ, C.; FERNÁNDEZ R.; BAPTISTA P. Metodología de la investigación. México: MacGraw-Hill. 2003.

${ }^{18}$ MARFELL-JONES, M. et al. International standard for anthropometric assessment. South Africa: ISAK: Potchefstroom, 2006.

${ }^{19}$ HEYWARD, V. Evaluación y prescripción del ejercicio. Barcelona: Paidotribo, 2001.

${ }^{20}$ FERNÁNDES FILHO, J. A prática da avaliação física. Rio de Janeiro: Shape, 1999.

${ }^{21}$ CHILE. Ministerio de Educación, Unidad de Currículum y Evaluación. Informe de resultados educación física. Santiago, 2011.

${ }^{22}$ MARTÍNEZ, E. Pruebas de aptitud física. Barcelona: Paidotribo, 2002.

${ }^{23}$ BLÁZQUEZ, D. Evaluar en Educación Física. Zaragoza: Inde, 1997.

${ }^{24}$ COSSIO-BOLANOS, M.; ARRUDA, M.; LEITE, D. Valoración del rendimiento físico de jóvenes futbolistas en función de la edad cronológica. Apunts Educación Física y Deportes, n. 106, p. 42-49, 2011.

${ }^{25}$ MASCARENHAS, L. et al. Comportamento do consumo máximo de oxigênio e da composição corporal durante o processo maturacional em adolescentes do sexo masculino participantes de treinamento de futebol. Revista Brasileira de Ciência e Movimiento, Brasília, v.14, n. 2, p. 49-56, 2006.

Conexões: revista da Faculdade de Educação Física da UNICAMP, Campinas, v. 12, n. 4, p. 1-22, out./dez. 2014. ISSN: 1983-9030 
${ }^{26} \mathrm{KOBAYASHI}, \mathrm{K}$. Aerobic power as related to body growth and training in japanese boys. Journal of Applied Physiology, v. 44, n. 5, p. 666-672, 1978.

${ }^{27}$ SEDANO, S.; CUADRADO, G.; REDONDO, J. C. Valoración de la influencia de la práctica del fútbol en la evolución de la fuerza, la flexibilidad y la velocidad en población infantil. Apunts. Educación Física y Deportes, n. 87, p. 54-63, 2007.

${ }^{28}$ CORREA, J. E. Determinación del perfil antropométrico y cualidades físicas de niños futbolistas de Bogotá. Revista Ciencias de la Salud, v 6, n. 2, p. 74-84, 2008.

${ }^{29}$ MIRELLA, R. Las nuevas metodologías del entrenamiento de la fuerza, la resistencia, la velocidad y la flexibilidad. Barcelona: Paidotribo, 2006.

${ }^{30}$ RIVERA, M.; AVELLA, F. Características antropométricas y fisiológicas de futbolistas portorriqueños. Archivos de Medicina del Deporte, v. 9, n. 35, p. 265-277, 1992.

${ }^{31}$ BRUGGEMANN, D.; ALBRECHT, D. Entrenamiento moderno del fútbol. Barcelona: Hispano Europea, 2000.

Recebido em: 12 maio 2014 Aceito em: 05 dez. 2014 Contato: cristiasn.luarte@uss.cl 